\title{
NOTES
}

\section{Unique Physical Adsorption of Proteins onto Double Stranded Stereocomplex Films Composed of Stereoregular Poly(methyl methacrylate)s}

\author{
Takeshi SerizawA $^{1}$, Keiko Yamashita, and Mitsuru AKashi ${ }^{2, \dagger}$ \\ Department of Nanostructured and Advanced Materials, Graduate School of Science and Engineering, \\ Kagoshima University, 1-21-40 Korimoto, Kagoshima 890-0065, Japan \\ ${ }^{1}$ Research Center for Advanced Science and Technology, The University of Tokyo, \\ 4-6-1 Komaba, Meguro-ku 153-8904, Japan \\ ${ }^{2}$ Department of Molecular Chemistry, Graduate School of Engineering, Osaka University, \\ 2-1 Yamada-oka, Suita 565-0871, Japan
}

(Received March 30, 2005; Accepted January 21, 2006; Published May 15, 2006)

KEY WORDS Stereocomplex / Poly(methyl methacrylate) / Protein / Adsorption / Double Strand / [doi:10.1295/polymj.38.503]

The nonspecific physical adsorption of proteins onto material surfaces is an essential event when non-biological materials initially come into contact with a biological atmosphere. ${ }^{1}$ Control over protein adsorption is therefore a necessary requirement for designing the surfaces of biomedical and biosensing devices. ${ }^{2}$ Although hydrophobicity as well as the charge of the material surfaces often modulate protein-adsorptive properties, the biophysical parameters governing protein adsorption are diverse. When parameters such as the chemical species of the materials or proteins used, the assembly structures of the material surfaces, and the adsorption media are simultaneously changed in each experiment, the mechanism of protein adsorption becomes more complicated. Accordingly, a fundamental analysis to separately determine the relative importance of each parameter using adequate surfaces as a model system is necessary for a clear understanding of protein adsorption.

We have previously demonstrated the stepwise, layer-by-layer fabrication of ultrathin stereocomplex films composed of stereoregular isotactic (it) and syndiotactic (st) poly(methyl methacrylate)s (PMMAs), followed by the alternate immersion of solid substrates into both solutions. ${ }^{3}$ In/on the films, the PMMAs formed a double-stranded helical structure, in/on which the it-PMMA was surrounded by a double length of st-PMMA based on structural fitting with van der Waals contact forces. ${ }^{4}$ This assembly process can also be applied to other stereocomplexes composed of stereoregular polymers of methacrylates ${ }^{5}$ and racemic poly(lactide)s. ${ }^{6}$ Regular assemblies at the macromolecular level demonstrated a novel macro- molecular recognition system using the bulk of the ultrathin polymer films, ${ }^{7}$ and led to the unexpected and rapid degradation of the poly(lactide)s. ${ }^{6 b}$ From the viewpoint of protein adsorption, the surfaces of these stereocomplex films possess great potential for analyzing the effects of the surface assembly structures on adsorption. We can readily compare the adsorptive properties between stereocomplex films and single component films conventionally prepared, because they have the same chemical structures and the similar surface hydrophobicity. ${ }^{3}$

Tests of adhesion using fibroblast cells in the absence of serum proteins on the surfaces of stereocomplex PMMA films pre-coated with physically adsorbed serum proteins such as cell-non-adhesive human serum albumin (HSA) and cell-adhesive fibronectin demonstrated more suppressed and enhanced cell adhesion, respectively, as compared to adhesion on the original stereocomplex film without pre-coating. ${ }^{8}$ These observations suggest that the denaturation of these proteins after physical adsorption was suppressed on the stereocomplex films, thus resulting in the expression of native bioactivity for both proteins. In fact, bovine serum albumin tended to adsorb onto stereocomplex films without denaturation. ${ }^{9}$ It was also noted that the cells adhered more strongly and proliferated better on the stereocomplex films as compared to the single component films, and to a conventionally prepared mixed film of PMMAs with stereocomplex stoichiometry. ${ }^{8}$

In the present study, the adsorption of proteins such as HSA, fibrinogen, and lysozyme onto ultrathin PMMA stereocomplex films was analyzed, and the

${ }^{\dagger}$ To whom correspondence should be addressed (E-mail: akashi@chem.eng.osaka-u.ac.jp). 
resulting data were compared with the adsorption onto single component PMMA films conventionally prepared. We discovered here a parameter of the surface nanostructure that affects protein adsorption. Furthermore, since hollow fiber membranes composed of cellulose mixed with PMMA stereocomplex are utilized for artificial dialysis, ${ }^{10}$ this research will provide new insights into the potential biomedical applications of PMMA stereocomplexes.

\section{EXPERIMENTAL}

It-PMMA $\left(M_{\mathrm{n}} 21900, M_{\mathrm{w}} / M_{\mathrm{n}} 1.16, m m: m r: r r=\right.$ 99:1:0 $)$ and st-PMMA $\left(M_{\mathrm{n}} 24200, M_{\mathrm{w}} / M_{\mathrm{n}}\right.$ 1.28, $m m: m r: r r=1: 9: 90)\left(M_{\mathrm{n}}, M_{\mathrm{w}}\right.$, and mm:mr:rr indicate average number, average weight molecular weights, and tacticity) were synthesized by conventional living anion polymerization. ${ }^{11} \mathrm{HSA}$ (fatty acid free) $\left(M_{\mathrm{w}}\right.$ 69000, $\mathrm{p} I 4.6)^{12}$ (pI indicates isoelectric point), fibrinogen $\left(M_{\mathrm{w}} 340000, \mathrm{p} I 5.5\right)$, and lysozyme $\left(M_{\mathrm{w}} 14000\right.$, $\mathrm{p} I$ 11.1 $)^{13}$ were purchased from Sigma. For the quantitative analysis of film fabrication and protein adsorption, a 9-MHz quartz crystal microbalance (QCM) substrate with a surface roughness of $1.7 \mathrm{~nm}$ (USI system, Japan), which detects the amount of polymer assembled on the electrodes from its frequency decrease, ${ }^{14}$ was utilized. For the $9-\mathrm{MHz}$ QCM substrate with gold electrodes ( $4.5 \mathrm{~mm}$ diameter), the frequency decrease $(-\Delta F)$ was converted to the amounts deposited $(\Delta m)$ using the following equation: $-\Delta F(\mathrm{~Hz})=$ $1.15 \times \Delta m(\mathrm{ng})$. The PMMA stereocomplex films were prepared according to previous reports. ${ }^{3,8,9}$ In brief, the substrate was alternately immersed into PMMA solutions of acetonitrile $\left(1.7 \mathrm{mg} \mathrm{mL}^{-1}\right)$ for (it-/st-PMMAs) ${ }_{6}$ cycles at $25^{\circ} \mathrm{C}$ for an immersion time of 15 min each, resulting in a mean film thickness of $11 \mathrm{~nm}$. The ratio of the amount of polymers assembled was estimated to be $1.9 \pm 0.4$ (st-/it-PMMAs, $\mathrm{w} / \mathrm{w}$ ), thereby indicating the formation of double strands during assembly. ${ }^{4}$ Homogeneous films composed of it- and st-PMMAs were prepared by conventional spin-casting (2000 rpm). Subsequently, proteins solubilized in phosphate-buffered saline (PBS) at the given concentrations were adsorbed onto the films at $37^{\circ} \mathrm{C}$ for the given incubation times, and then frequency decreases were measured to obtain the amounts of proteins adsorbed. $-\Delta F$ was measured in air in all experiments.

The mean roughness of the film surfaces in an area of $2 \times 2 \mu \mathrm{m}$ was estimated by atomic force microscopy (AFM) (Nanoscope III), and the thickness of the films was analyzed by a scratching mode. Attenuated total reflection (ATR) spectra (Specrum One, Perkin Elmer) were obtained using a refractive surface of the QCM gold electrode. The interferograms were co-added 50 times, and Fourier transformed at a resolution of $4 \mathrm{~cm}^{-1}$.

\section{RESULTS AND DISCUSSION}

We prepared three types of PMMA films composed of a double stranded stereocomplex, homogeneous it-, and st-PMMAs on the substrates. Since the chemical structure is the same, the effects of the assembly structures as well as tacticities on the amount of protein adsorbed were analyzed. Figure 1 shows the adsorption isotherms of selected proteins onto these films. The amounts of proteins adsorbed increased with increasing concentrations of proteins, and saturated at above approximately $3 \mathrm{mg} \mathrm{mL}^{-1}$ in all cases. From the isotherms, it was clearly observed that the amounts adsorbed onto the stereocomplex film were greater
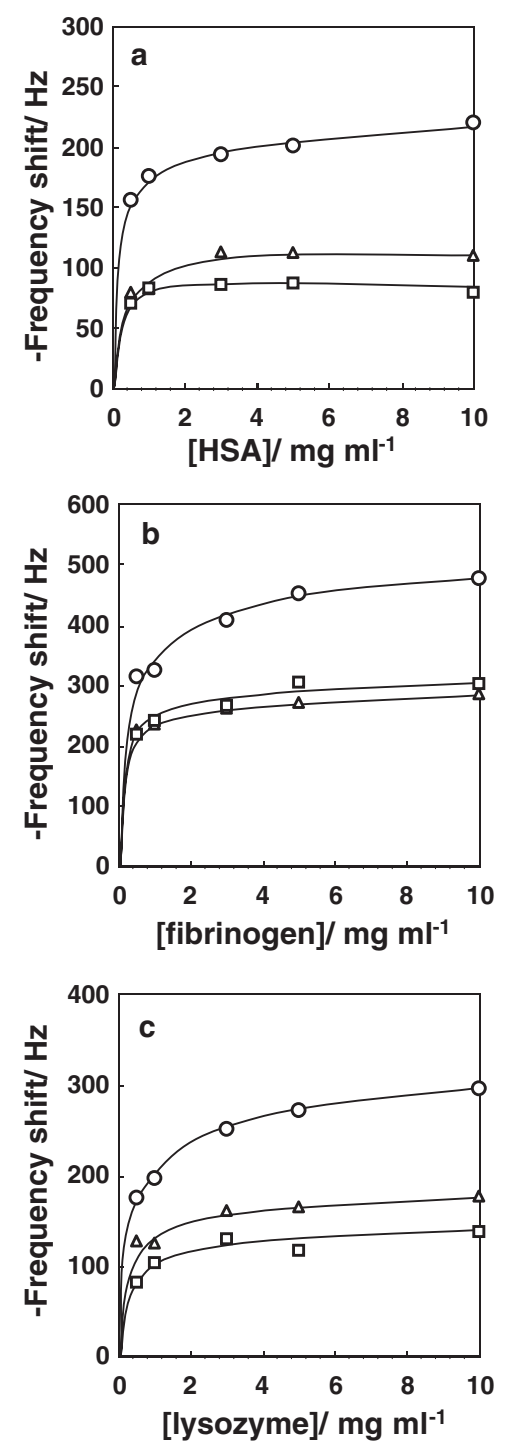

Figure 1. Adsorption isotherms of (a) HSA, (b) fibrinogen, and (c) lysozyme. Circle, triangle, and square symbols indicate the adsorption onto the stereocomplex, it-, and st-PMMA films, respectively. 
than those onto the component films, suggesting a different mode of protein adsorption onto the stereocomplex film. The mean roughness of the stereocomplex film, homogeneous it- and st-PMMA films were estimated to be 5.4, 11, and $12 \mathrm{~nm}$ by the AFM surface analysis, suggesting that the surface area of the films can not explain the adsorption onto the stereocomplex film. In order to discuss the adsorption quantitatively, the isotherms were fitted to a monolayer adsorption process expressed by the following Langmuir plot equation:

$$
\frac{[\text { protein }]}{\Delta F}=\frac{1}{\Delta F_{\max }}[\text { protein }]+\frac{1}{\Delta F_{\max } K_{\mathrm{ads}}}
$$

where $\Delta F_{\max }$ is the maximum frequency decrease corresponding to the maximum amount of proteins adsorbed and $K_{\text {ads }}$ is the apparent adsorption constant. The isotherms fitted well to the plot, with a coefficient of variation of $>0.99$. The adsorption parameters were then estimated and summarized in Table I.

HSA and fibrinogen have size dimensions of $12 \times$ $2.7 \times 2.7$ and $47 \times 5 \times 5 \mathrm{~nm}$, respectively. ${ }^{12,13}$ Therefore, end-on and side-on adsorption modes for monolayer coverage may exist, resulting in the greatest and smallest adsorption amounts, respectively. The adsorption amounts expected for the two modes have already been estimated to be 0.9 and $0.25 \mu \mathrm{g} \mathrm{cm}^{-2}$ for HSA, and 1.7 and $0.18 \mu \mathrm{g} \mathrm{cm}^{-2}$ for fibrinogen, respectively. ${ }^{15}$ The present data ranged between these values, except for the adsorption amount of HSA onto the stPMMA film, indicating monolayer coverage for the proteins adsorbed. Interestingly, a greater amount of HSA and fibrinogen with the end-on mode was adsorbed onto the stereocomplex film as compared to the other homogeneous films. In terms of stereoregularity, the difference between the it- and st-PMMA films was relatively small, and the adsorption amounts were dependent on the proteins adsorbed. These observations suggest that the formation of double strands of PMMA

Table I. Adsorption Parameters of Proteins

\begin{tabular}{lccc}
\hline \multicolumn{1}{c}{ Film } & Protein & $\begin{array}{c}\Delta m_{\max ^{\mathrm{a}}} / \\
\mu \mathrm{g} \mathrm{cm}^{-2}\end{array}$ & $\begin{array}{c}K_{\mathrm{ads}}^{\mathrm{b}} / \\
\times 10^{5} \mathrm{M}^{-1}\end{array}$ \\
\hline it-PMMA & & 0.27 & 3.75 \\
st-PMMA & HSA & 0.17 & 5.90 \\
stereocomplex & & 0.60 & 1.90 \\
it-PMMA & & 0.68 & 1.64 \\
st-PMMA & fibrinogen & 0.69 & 1.38 \\
stereocomplex & & 1.34 & 0.82 \\
it-PMMA & & $(0.41)^{\mathrm{c}}$ & - \\
st-PMMA & lysozyme & $(0.29)^{\mathrm{c}}$ & - \\
stereocomplex & & $(0.79)^{\mathrm{c}}$ & - \\
\hline
\end{tabular}

${ }^{a}$ Maximum adsorption amount. ${ }^{b}$ apparent adsorption constant. ${ }^{\mathrm{c}}$ Adsorption amount at $10 \mathrm{mg} \mathrm{mL}^{-1}$. at the film-water interface significantly affected protein adsorption. On the other hand, the amount of lysozyme adsorbed was also greatest onto the stereocomplex film, although lysozyme $(4 \times 3 \times 3 \mathrm{~nm} \text { in size })^{13}$ is almost spherical in shape. Lysozyme is known to form multilayers at greater concentrations. ${ }^{13}$ In fact, the present amounts of lysozyme adsorbed indicate the formation of multilayers. These observations suggest that the lysozyme multilayers tend to be formed more readily on the stereocomplex film, also suggesting a different mechanism of protein adsorption between the stereocomplex and homogeneous films. It should be noted that the present adsorption cannot be explained by the hydrophobicity of the film surfaces, since the static contact angles using water droplets were similar and ranged between 63-73 degrees. ${ }^{3}$ Hydration of the stereocomplex film might be different from those of homogeneous films, and will be analyzed by using the surface Raman scattering.

For the adsorptions of HSA and lysozyme, the apparent adsorption constants were estimated from the Langmuir plot. In both cases, the constants onto the stereocomplex film were the smallest as compared to the others, indicating that the proteins were more weakly adsorbed onto the stereocomplex film. Accordingly, greater amounts of proteins were adsorbed onto the stereocomplex film on the basis of weaker interactions as compared to the homogeneous component films. Since the interaction force between the stereocomplex film and the proteins were relatively weaker, the proteins might interact effectively with each other during the adsorption, thus resulting in the end-on mode, in which the proteins nestle up to each other on the film surface. The formation of greater multilayers of lysozyme adsorbed onto the stereocomplex film might also be interpreted by extending this mechanism.

Using HSA, we analyzed the adsorption in more detail. The monolayer coverage of HSA adsorbed onto the stereocomplex film at 0.5 and $5 \mathrm{mg} \mathrm{mL}^{-1}$ was also supported by an analysis of the surface roughness by AFM, in which the initial film roughness $(5.4 \mathrm{~nm})$ increased slightly to 5.8 and $6.1 \mathrm{~nm}$, respectively. Furthermore, the monolayer thickness of HSA adsorbed at $5 \mathrm{mg} \mathrm{mL}^{-1}$ onto the stereocomplex film and a homogeneous it-PMMA film were analyzed by the difference between the film thickness and the HSA-adsorbed total thickness using the AFM scratching mode. The thicknesses were estimated to be 10 and $5 \mathrm{~nm}$, respectively, also supporting the hypothesis that greater amounts of HSA were adsorbed with an endon mode. For analyzing the extent of the denaturation of the HSA adsorbed, ATR spectra in the region of the amide absorption bands, which reflect the amounts of secondary ordered structures such as $\alpha$-helices and $\beta$ - 


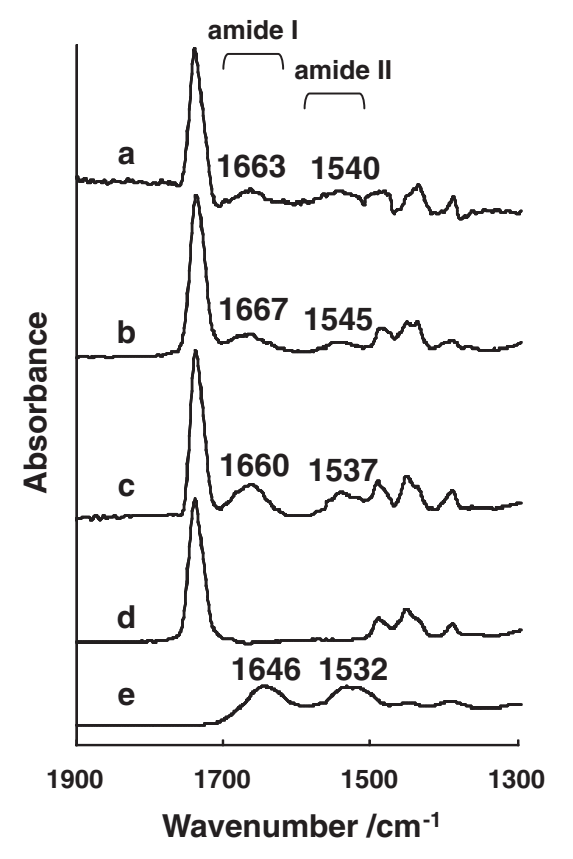

Figure 2. ATR spectra of HSA adsorbed onto (a) it-PMMA film, (b) st-PMMA film, and (c) stereocomplex film, of (d) stereocomplex film, and (e) HSA powder.

sheets, were obtained. As shown in Figure 2, the peak shifts against those of HSA powder were more attenuated on the stereocomplex film, thus indicating that the denaturation of HSA was more attenuated on the complex film. It is noted that the carbonyl vibration bands for stereocomplex-forming PMMA, of which the peak position had been utilized for evidence for complex formation, was not changed after the adsorption of HSA. This suggests that the assembly structure of PMMAs was conserved after the adsorption. As a consequence, the double strands affected not only the adsorption mechanism but also the denaturation of proteins.

The physical adsorption of proteins such as HSA, fibrinogen, and lysozyme onto PMMA stereocomplex films and single component homogeneous films was evaluated. QCM analysis revealed that greater amounts of proteins with smaller adsorption constants were adsorbed onto the stereocomplex film as compared to the homogeneous films. The formation of monolayers was observed for HSA and fibrinogen, whereas lysozyme formed multilayers. ATR spectra of the HSA adsorbed showed less denaturation when adsorbed onto the stereocomplex film. As a consequence, the present study demonstrated that the surface assembly nanostructure is an important factor for governing protein adsorption. Further research is necessary to confirm this concept using other regularly prepared ultrathin films.
Acknowledgment. Authors appreciate technological support of living anionic polymerization by Emeritus Prof. Koichi Hatada (Osaka University) and Prof. Tatsuki Kitayama (Osaka University).

\section{REFERENCES}

1. a) American Chemical Society, "Protein at Interfaces: Physicochemical and Biochemical Studies," T. A. Horbett and J. L. Brash, Ed., Washington DC, 1987.

b) American Chemical Society, "Protein at Interfaces II: Fundamentals and Applications," J. L. Brash and T. A. Horbett, Ed., Washington DC, 1995.

2. a) J. D. Andrade and V. Hlady, Adv. Polym. Sci., 79, 1 (1986).

b) B. D. Ratner, F. J. Hoffmann, J. E. Schoen, and F. Lemons, "Biomaterials Science: An Introduction to Materials in Medicine," Academic Press, New York, 1996.

c) E. Ostumi, R. G. Chapman, M. N. Liang, G. Meluleni, G. Pier, D. E. Ingber, and G. M. Whitesides, Langmuir, 17, 6336 (2001).

3. T. Serizawa, K.-I. Hamada, T. Kitayama, N. Fujimoto, K. Hatada, and M. Akashi, J. Am. Chem. Soc., 122, 1891 (2000)

4. E. Schomaker and G. Challa, Macromolecules, 22, 3337 (1989).

5. a) T. Serizawa, K.-I. Hamada, T. Kitayama, N. Fujimoto, K. Hatada, and M. Akashi, Langmuir, 16, 7112 (2000).

b) K.-I. Hamada, T. Serizawa, T. Kitayama, N. Fujimoto, K. Hatada, and M. Akashi, Langmuir, 17, 5513 (2001).

6. a) T. Serizawa, H. Yamashita, T. Fujiwara, Y. Kimura, and M. Akashi, Macromolecules, 34, 1996 (2001).

b) T. Serizawa, Y. Arikawa, K.-I. Hamada, H. Yamashita, T. Fujiwara, Y. Kimura, and M. Akashi, Macromolecules, 36, 1762 (2003).

7. a) T. Serizawa, K.-I. Hamada, T. Kitayama, and M. Akashi, Angew. Chem., Int. Ed., 42, 1118 (2003).

b) T. Serizawa, K.-I. Hamada, and M. Akashi, Nature, 429, 52 (2004).

8. T. Serizawa, K. Yamashita, and M. Akashi, J. Biomater. Sci., Polym. Ed., 15, 511 (2004).

9. K.-I. Hamada, K. Yamashita, T. Serizawa, T. Kitayama, and M. Akashi, J. Polym. Sci., Part A: Polym. Chem., 41, 1807 (2003).

10. B. Bikson, J. K. Nelson, and N. Muruganandam, J. Membr. Sci., 94, 313 (1994).

11. a) K. Hatada, K. Ute, Y. Okamoto, and T. Kitayama, Polym. J., 18, 1037 (1986).

b) T. Kitayama, T. Shinozaki, T. Sakamoto, M. Yamamoto, and K. Hatada, Makromol. Chem, 15, 167 (1989).

12. C. A. Hynes and W. Norde, Colloid Surf. B, 2, 517 (1994).

13. J. Kim and G. A. Somorjai, J. Am. Chem. Soc., 125, 3150 (2003).

14. G. Sauerbrey, Z. Phys., 155, 206 (1959).

15. A. Baszkin and D. J. Lyman, J. Biomed. Mater. Res., 14, 393 (1980). 